\title{
Comparative transcriptional profiling of medullary and cortical thymic cell types identifies epigenetic and transcription factors that uniquely define the MTEChi and cTEC lineages
}

Shahan Mamoor

shahanmamoor@gmail.com

San Diego, CA 92101

The thymus has the unique ability to impart lymphocytes in trans the concept of self-tolerance by negative selection and to enforce positive selection of lymphocytes that express optimal Tcell receptors $(1,2)$. The thymus possesses a cortex and a medulla (4), and epithelial cells known as mTEC and CTEC are intimately involved in these processes (5). Here I compared the transcriptomes of mTEChi and cTEC (6) using global differential gene expression analysis. I present a series of epigenetic molecules and transcription factors that represent the most significant differences in the global gene expression profiles between these two cell types.

Keywords: thymus, medullary epithelial cells, cortical epithelial cells, mTEChi, cTEC, Utf1, Foxe1, Hoxd11, Fezf2, Myb, Arid4a, Ascl4, Baz2a, Rbmx, Rbm47, R3hcc1, Zfc3h1, Zcchc7, autoimmunity, transplant medicine 


\section{Introduction}

The thymus has the remarkable ability to provide lymphocytes the instructions for two essential processes in the human immune system known as negative and positive selection (1, 2). In negative selection, developing lymphocytes are displayed self-antigen, peptides that represent antigens that otherwise would be restricted to the periphery, known as tissuerestricted antigen (TRA) by presentation on the class II major histocompatibility complex (MHCII) on mTEChi cells (6). TRA expression in mTEChi, cells that express high levels of MHC-II, is in large part dependent on the autoimmune regulator Aire (7) and lymphocytes whose T-cell receptors (TCRs) recognize self-antigen displayed on mTEChi MHC-II are deleted, or negatively selected (8). This process enables the immune system to remove any lymphocytes that would otherwise go on to enter the periphery and attack self cells, tissues and organs rather than pathogens (8). Positive selection occurs in the cortex of the thymus in cTEC $(9,10)$ and in this process, TCR that display an optimal binding affinity to peptide-MHC complexes displayed on the cTEC surface are chosen to survive, or positively selected (11-13). Developing lymphocytes whose TCR display binding affinity that is relatively strong or relatively weak are either deleted or left to die in a process known as anergy or death by neglect $(1,2,14,15)$.

The combined processes of positive and negative selection, occurring by interactions with the mTEC and CTEC of the thymus, are essential for the generation of an adaptive immune system that has the ability to defend the human body against pathogens while also preventing dangerous autoimmune pathology $(8,16-18)$. Understanding the transcriptional behaviors of both $\mathrm{mTEC}$ and CTEC are critical for complete understanding of how positive and negative selection occur, for the discovery of novel pathways to combat infectious and autoimmune diseases, and for the generation of novel tools and techniques to treat transplant rejection (19-21). In this study, we compared the total gene expression profiles, or transcriptomes, of mTEChi and cTEC using a public dataset (3) to determine that most significant transcriptional changes between these two cell types. This analysis revealed that multiple transcription factors and epigenetic molecules were represented among the most differentially expressed 
genes in the mTEChi and CTEC transcriptomes. Remarkably, multiple RNA-binding proteins were also found to be among the genes whose expression was most different between these two cell types.

\section{Methods}

Dataset GSE89965 (3) was utilized for this differential gene expression analysis, performed using GEO2R. This public dataset was generated by investigators (3) using microarray (Affymetrix Mouse Gene 1-ST array) analysis of Aire-GFP, EpCAM+Ly51+ cells (cTEC), and Aire-GFP Epcam+MHC-II(hi) cells (mTEChi); $n=4$ from mTEChi and $n=3$ from cTEC, from C57BI/6 Adig mice 6 weeks of age (adult). No adjustment was made to $p$-values, and there was no log-transformation applied to the data. The NCBI generated category of platform annotation was used. An unpaired two-tailed t-test with Welch's correction was utilized to determine the statistical significance of the RNA expression values between each cell type.

\section{Results}

We performed global differential gene expression profiling of the mTEChi and cTEC transcriptomes (3) to understand in a systematic fashion the transcriptional behavior of these immunologically important thymic cells types and to determine the most significant transcriptional changes between medullary and cortical thymic epithelium, cells required for the processes of positive and negative selection (1, 2, 4, 5, 8-10).

\section{A series of differentially expressed transcription factors that uniquely define the mTEChi and CTEC lineages.}

Five transcription factors were among the most differentially expressed genes when comparing the transcriptomes of $\mathrm{mTEC}^{\text {hi }}$ and cTEC. Three were expressed at higher levels in mTEChi, while two were expressed at higher levels in cTEC. These included Utf1, Foxe1, Fezf2, Hoxd11, and Myb. 
Utf1, undifferentiated embryonic cell transcription factor 1, was the 2nd most differentially expressed gene globally when comparing the mTEChi and cTEC transcriptomes (Table 1; $\mathrm{p}=2.42 \mathrm{E}-08$ ). Utf1 expression was significantly higher in $\mathrm{mTEChi}$ than in cTEC (Figure $1 ; p<0.0001)$.

Foxe1, forkhead box E1, was the 12th most differentially expressed gene globally when comparing the mTEChi and cTEC transcriptomes (Table 1; $p=9.17 E-07)$. Foxe1 expression was significantly higher in cTEC than in mTEChi (Figure $1 ; p=0.0001)$.

Fezf2, Fez family zinc finger 2, was the 26th most differentially expressed gene globally when comparing the mTEChi and cTEC transcriptomes (Table 1; $p=1.51 E-06$ ). Fezf2 expression was significantly higher in mTEChi than in cTEC (Figure 1; $p=0.0001$ ).

Hoxd11, homeobox D11, was the 64th most differentially expressed gene globally when comparing the mTEChi and cTEC transcriptomes (Table 1; $\mathrm{p}=5.19 \mathrm{E}-06$ ). Hoxd11 expression was significantly higher in cTEC than in mTEChi (Figure $1 ; p=0.0015)$.

Myb, myeloblastosis oncogene, was the 15th most differentially expressed gene globally when comparing the mTEChi $^{\text {hi }}$ and cTEC transcriptomes (Table $\left.1 ; p=2.34 E-05\right)$. Myb expression was significantly higher in mTEChi than in cTEC (Figure $1 ; p=0.0005$ ).

\section{A series of epigenetic molecules that are among the most significantly differentially expressed genes when comparing the $\mathrm{mTECh}^{\mathrm{hi}}$ and cTEC transcriptomes.}

Six epigenetic molecules were represented among the messages whose expression was most different in the global transcription profiles of mTEChi and cTEC. All but one were expressed at higher levels in mTEChi. These included Med12I, Rbpj, Sirt1, Baz2a, Ascl4, and Arid4a.

Med12I, mediator complex subunit 12-like, was the 54th most differentially expressed gene globally when comparing the mTEChi and cTEC transcriptomes (Table $1 ; p=4.37 \mathrm{E}-06$ ). Med12l expression was significantly higher in mTEChi than in cTEC (Figure 2; $p<0.0001$ ).

Rbpj, recombination signal binding protein for immunoglobulin kappa $\mathrm{J}$ region, was the 74th most differentially expressed gene globally when comparing the mTEChi and cTEC 
transcriptomes (Table 1; $\mathrm{p}=6.08 \mathrm{E}-06$ ). Rbpj expression was significantly higher in mTEChi than in cTEC (Figure $2 ; p=0.0003$ ).

Sirt 1 , sirtuin 1 , was the 125th most differentially expressed gene globally when comparing the mTEChi and cTEC transcriptomes (Table 1; $p=1.16 E-05)$. Sirt1 expression was significantly higher in mTEChi than in cTEC (Figure $2 ; p=0.0006$ ).

Baz2a, bromodomain adjacent to zinc finger domain, 2A, was the 76th most differentially expressed gene globally when comparing the mTEChi and cTEC transcriptomes (Table 1; $p=6.27 E-06)$. Baz2a expression was significantly higher in mTEChi than in cTEC (Figure 2; $\mathrm{p}=0.0025)$.

Ascl4, achaete-scute family bHLH transcription factor 4, was the 152nd most differentially expressed gene globally when comparing the mTEChi and cTEC transcriptomes (Table 1; $p=1.5 E-05)$. Ascl4 expression was significantly higher in cTEC than in mTEChi (Figure $2 ; p=0.0026$.

Arid4a, AT rich interactive domain 4A (RBP1-like), was the 172nd most differentially expressed gene globally when comparing the mTEC $^{\text {hi }}$ and CTEC transcriptomes (Table 1; $\mathrm{p}=1.81 \mathrm{E}-05)$. Arid4a expression was significantly higher in mTEChi than in cTEC (Figure 2; $p=0.0001)$.

\section{Multiple RNA-binding proteins uniquely define the $\mathrm{mTEC}^{\mathrm{hi}}$ lineage and are among the most differentially expressed genes when comparing the $\mathrm{mTEChi}$ and cTEC transcriptomes.}

Interestingly, five separate RNA binding proteins were among the most differentially expressed genes when comparing transcriptomes of mTEChi and cTEC. All but one were expressed at significantly higher levels in mTEChi. These included Rbmx, Rbm47, R3hcc1, Zfc3h1, and Zcchc7.

Rbmx, RNA binding motif protein, $\mathrm{X}$ chromosome, was the 61st most differentially expressed gene globally when comparing the mTEChi and CTEC transcriptomes (Table 1; 
$\mathrm{p}=5.03 \mathrm{E}-06$ ). Rbmx expression was significantly higher in $\mathrm{mTEChi}$ than in cTEC (Figure 3; $\mathrm{p}<0.0001)$.

Rbm47, RNA binding motif protein 47, was the 92nd most differentially expressed gene globally when comparing the mTEChi and cTEC transcriptomes (Table 1; $p=8.33 E-06)$. Rbm47 expression was significantly higher in $\mathrm{mTEC}^{\text {hi }}$ than in cTEC (Figure 3; $\mathrm{p}=0.0002$ ).

R3hcc1, R3H domain and coiled-coil containing 1, was the 137th most differentially expressed gene globally when comparing the mTEChi and cTEC transcriptomes (Table 1; $p=1.3 E-05)$. R3hcc1 expression was significantly higher in cTEC than in mTEChi (Figure 3; $\mathrm{p}<0.0001)$.

Zfc3h1, zinc finger, C3H1-type containing, was the 148th most differentially expressed gene globally when comparing the mTEChi and cTEC transcriptomes (Table $1 ; p=1.44 E-05$ ). Zfc3h1 expression was significantly higher in mTEChi than in cTEC (Figure 3; $p<0.0001$ ).

Zcchc7, zinc finger, $\mathrm{CCHC}$ domain containing 7, was the 221st most differentially expressed gene globally when comparing the mTEChi and cTEC transcriptomes (Table 1; $\mathrm{p}=2.6 \mathrm{E}-05)$. Zcchc7 expression was significantly higher in $\mathrm{mTEC}^{\text {hi }}$ than in cTEC (Figure 3; $p=0.0003)$.

\section{Two zinc-finger containing proteins uniquely define the mTEChi and cTEC lineages and are among the most differentially expressed genes when comparing the MTEChi and cTEC transcriptomes.}

Of the messages whose expression level was most different between the transcriptomes of $\mathrm{mTEC}^{\text {hi }}$ and cTEC, two zinc finger proteins were represented, and one was expressed at higher levels in mTEChi while the other was expressed at higher levels in cTEC.

Zfp395, zinc finger protein 395, was the 17th most differentially expressed gene globally when comparing the mTEChi and cTEC transcriptomes (Table 1; p=1.22E-06). Zfp395 expression was significantly higher in cTEC than in mTEChi (Figure 4; $p=0.0012$ ). 
Zfp397, zinc finger protein 397, was the 35th most differentially expressed gene globally when comparing the mTEChi and cTEC transcriptomes (Table 1; $p=2.57 E-06)$. Zfp397 expression was significantly higher in mTEChi than in cTEC (Figure 4; $p<0.0001$ ).

\section{Discussion}

In this study, we compared the mTEChi and cTEC transcriptomes (3) using global differential gene expression profiling. We discovered five transcription factors, Utf1, Foxe1, Fezf2, Hoxd11 and Myb, six epigenetic and DNA-binding molecules Med12l, Rbpj, Sirt1, Baz2a, Ascl4 and Arid4a, five RNA-binding proteins, Rbmx, Rbm47, R3hcc1, Zfc3h1 and Zcchc7, and two zinc-finger containing proteins, Zfp395 and Zfp397, among the genes whose expression was most different when considering the total transcriptomes of both cell types.

Many of these molecules are not well-characterized. Understanding the function of these molecules in health and how they might malfunction in disease is important for future work that might manipulate these gene products through small molecules, antibodies or gene therapy in order to specifically target autoimmune pathologies and transplant rejection. Generation of mouse models with specific knockouts of these differentially expressed genes in the thymus using B5t-Cre (22-24) should be prioritized in order to begin to understand the function of these gene products in the thymus in vivo such that the relevant pathways can be targeted for generation of novel, targeted therapeutics to treat autoimmunity and transplant rejection. 


\section{References}

1. Surh, C.D. and Sprent, J., 1994. T-cell apoptosis detected in situ during positive and negative selection in the thymus. Nature, 372(6501), p.100.

2. Blackman, M., Kappler, J. and Marrack, P., 1990. The role of the T cell receptor in positive and negative selection of developing T cells. Science, 248(4961), pp.1335-1341.

3. Jakub Abramson, Immunology, Weizmann Institute; PRJNA354356; GEO: GSE89965.

4. Rodewald, Hans-Reimer, Sabine Paul, Corinne Haller, Horst Bluethmann, and Carmen Blum. "Thymus medulla consisting of epithelial islets each derived from a single progenitor." Nature 414, no. 6865 (2001): 763.

5. Rossi, S.W., Jenkinson, W.E., Anderson, G. and Jenkinson, E.J., 2006. Clonal analysis reveals a common progenitor for thymic cortical and medullary epithelium. Nature, 441 (7096), p.988.

6. Hinterberger, M., Aichinger, M., Da Costa, O.P., Voehringer, D., Hoffmann, R. and Klein, L., 2010. Autonomous role of medullary thymic epithelial cells in central CD4+ T cell tolerance. Nature immunology, 11(6), p.512.

7. Anderson, M.S., Venanzi, E.S., Klein, L., Chen, Z., Berzins, S.P., Turley, S.J., Von Boehmer, H., Bronson, R., Dierich, A., Benoist, C. and Mathis, D., 2002. Projection of an immunological self shadow within the thymus by the aire protein. Science, 298(5597), pp. 1395-1401.

8. Liston, A., Lesage, S., Wilson, J., Peltonen, L. and Goodnow, C.C., 2003. Aire regulates negative selection of organ-specific T cells. Nature immunology, 4(4), p.350.

9. Laufer, T.M., DeKoning, J., Markowitz, J.S., Lo, D. and Glimcher, L.H., 1996. Unopposed positive selection and autoreactivity in mice expressing class II MHC only on thymic cortex. Nature, 383(6595), p.81.

10. Bill, J. and Palmer, E., 1989. Positive selection of CD4+ T cells mediated by MHC class IIbearing stromal cell in the thymic cortex. Nature, 341(6243), p.649.

11. Moran, A.E. and Hogquist, K.A., 2012. T-cell receptor affinity in thymic development. Immunology, 135(4), pp.261-267.

12. Klein, L., Hinterberger, M., Wirnsberger, G. and Kyewski, B., 2009. Antigen presentation in the thymus for positive selection and central tolerance induction. Nature Reviews Immunology, 9(12), p.833.

13. Stefanski, H.E., Mayerova, D., Jameson, S.C. and Hogquist, K.A., 2001. A low affinity TCR ligand restores positive selection of CD8+ T cells in vivo. The Journal of Immunology, 166(11), pp.6602-6607.

14. von Boehmer, H., 1994. Positive selection of lymphocytes. Cell, 76(2), pp.219-228. 
15. Palmer, E., 2003. Cell death and immunity: Negative selection-clearing out the bad apples from the T-cell repertoire. Nature Reviews Immunology, 3(5), p.383.

16. Kretz-Rommel, A. and Rubin, R.L., 2000. Disruption of positive selection of thymocytes causes autoimmunity. Nature medicine, 6(3), p.298.

17. Sakaguchi, N., Takahashi, T., Hata, H., Nomura, T., Tagami, T., Yamazaki, S., Sakihama, T., Matsutani, T., Negishi, I., Nakatsuru, S. and Sakaguchi, S., 2003. Altered thymic T-cell selection due to a mutation of the ZAP-70 gene causes autoimmune arthritis in mice. Nature, 426(6965), p.454.

18. Von Boehmer, H. and Melchers, F., 2010. Checkpoints in lymphocyte development and autoimmune disease. Nature immunology, 11(1), p.14.

19. Bonomo, A. and Matzinger, P., 1993. Thymus epithelium induces tissue-specific tolerance. Journal of Experimental Medicine, 177(4), pp.1153-1164.

20. Posselt, A.M., Barker, C.F., Tomaszewski, J.E., Markmann, J.F., Choti, M.A. and Naji, A., 1990. Induction of donor-specific unresponsiveness by intrathymic islet transplantation. Science, 249(4974), pp.1293-1295.

21. Jordan, R.K., Robinson, J.H., Hopkinson, N.A., House, K.C. and Bentley, A.L., 1985. Thymic epithelium and the induction of transplantation tolerance in nude mice. Nature, 314(6010), p.454.

22. Murata, S., Sasaki, K., Kishimoto, T., Niwa, S.I., Hayashi, H., Takahama, Y. and Tanaka, K., 2007. Regulation of CD8+ T cell development by thymus-specific proteasomes. Science, 316(5829), pp.1349-1353.

23. Ohigashi, I., Zuklys, S., Sakata, M., Mayer, C.E., Zhanybekova, S., Murata, S., Tanaka, K., Holländer, G.A. and Takahama, Y., 2013. Aire-expressing thymic medullary epithelial cells originate from $\beta 5 \mathrm{t}$-expressing progenitor cells. Proceedings of the National Academy of Sciences, 110(24), pp.9885-9890.

24. Ohigashi, I., Zuklys, S., Sakata, M., Mayer, C.E., Hamazaki, Y., Minato, N., Hollander, G.A. and Takahama, Y., 2015. Adult thymic medullary epithelium is maintained and regenerated by lineage-restricted cells rather than bipotent progenitors. Cell reports, 13(7), pp. 1432-1443. 

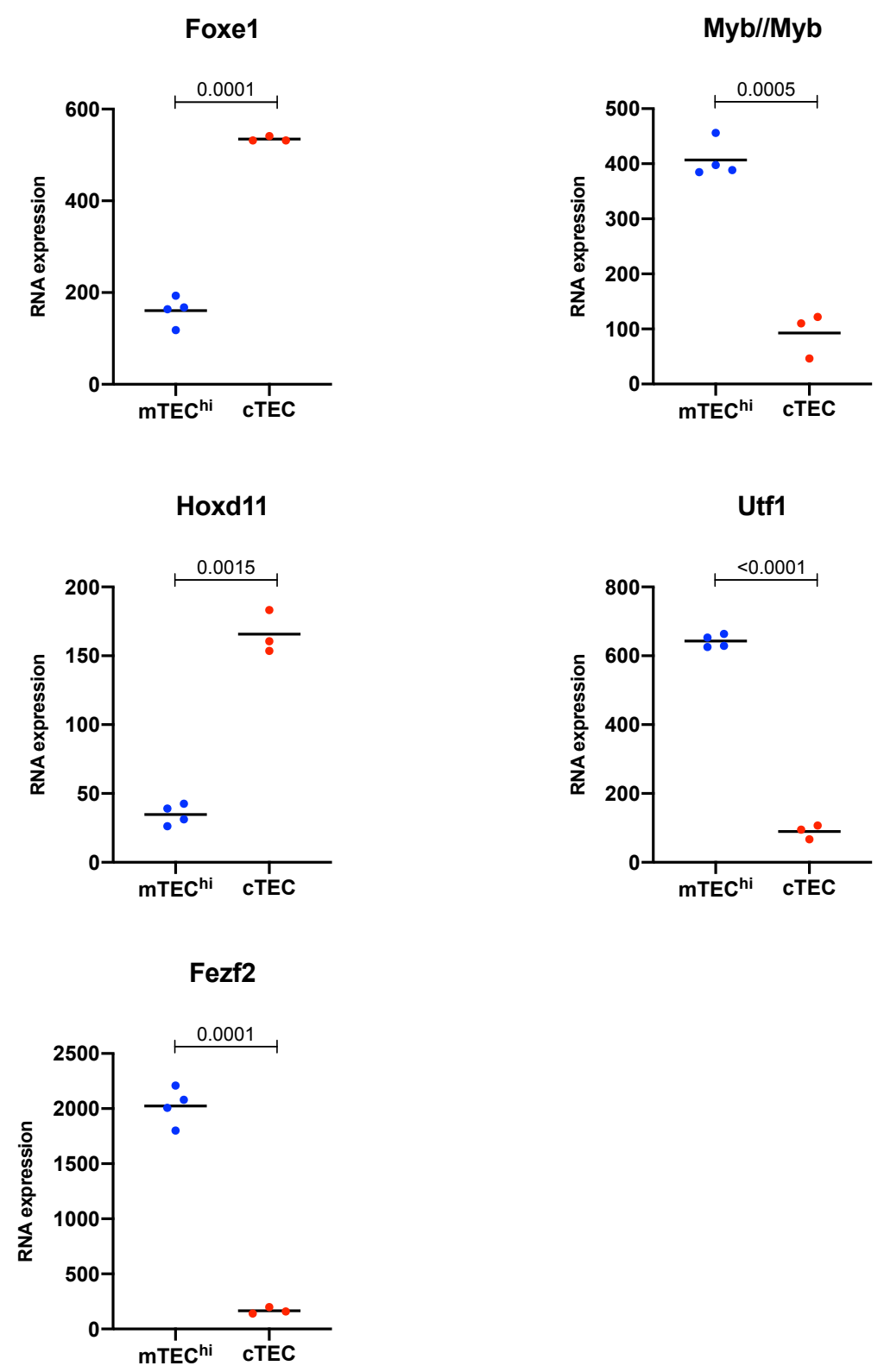

Figure 1: A series of differentially expressed transcription factors that unique define the mTEChi and CTEC lineages.

The expression of each differentially expressed gene is represented graphically, with the mean RNA expression value denoted by a horizontal black bar: mTEChi, $n=4$; cTEC, $n=3$. The statistical significance of the difference between the mean values between each cell type was calculated using a two-tailed unpaired t-test with Welch's correction, shown as a $p$-value on each graph. 

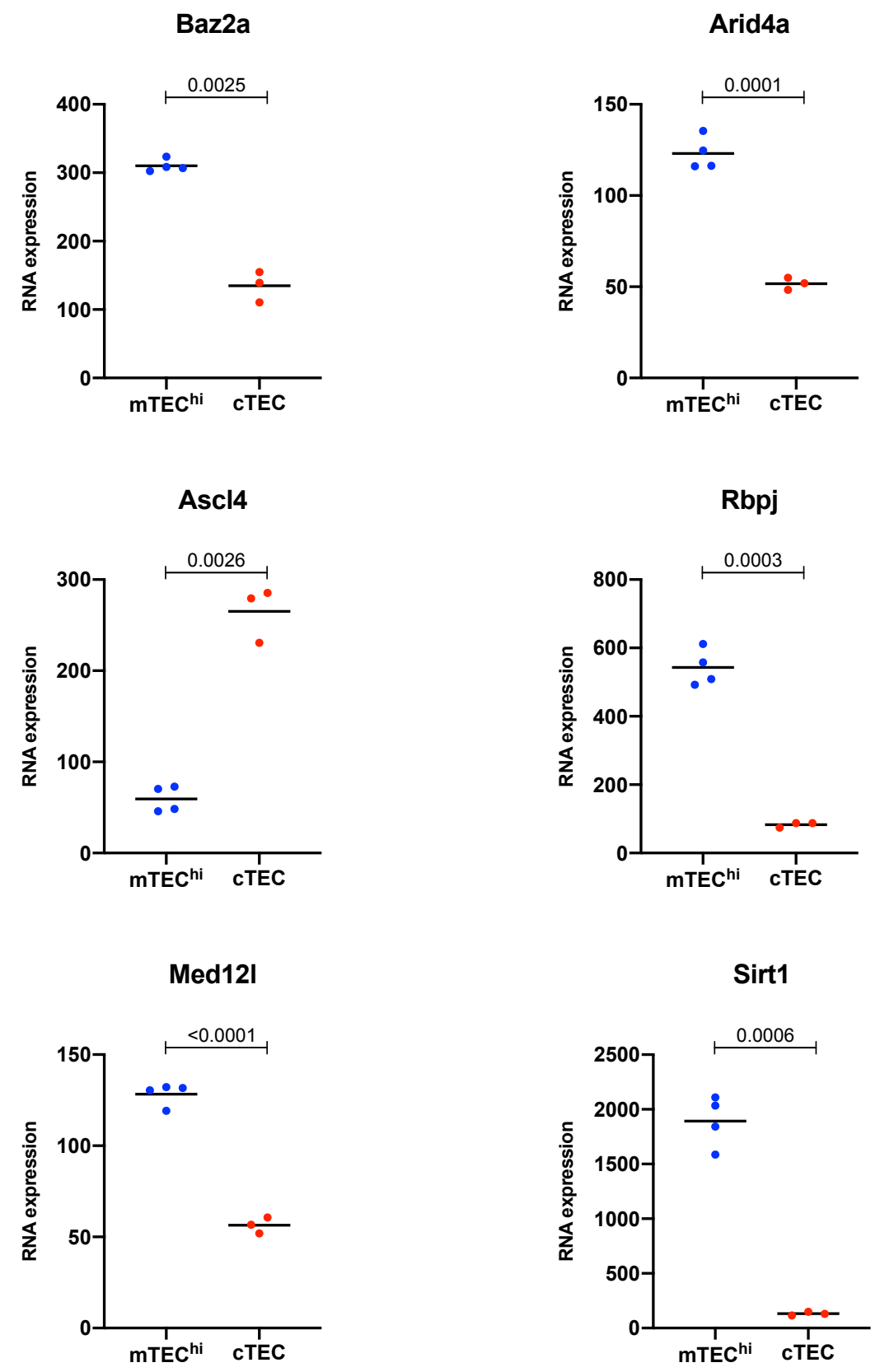

Figure 2: A series of epigenetic molecules that are among the most significantly differentially expressed genes when comparing the MTEChi and cTEC transcriptomes.

The expression of each differentially expressed gene is represented graphically, with the mean RNA expression value denoted by a horizontal black bar: mTEChi, $n=4$; cTEC, $n=3$. The statistical significance of the difference between the mean values between each cell type was calculated using a two-tailed unpaired t-test with Welch's correction, shown as a $p$-value on each graph. 

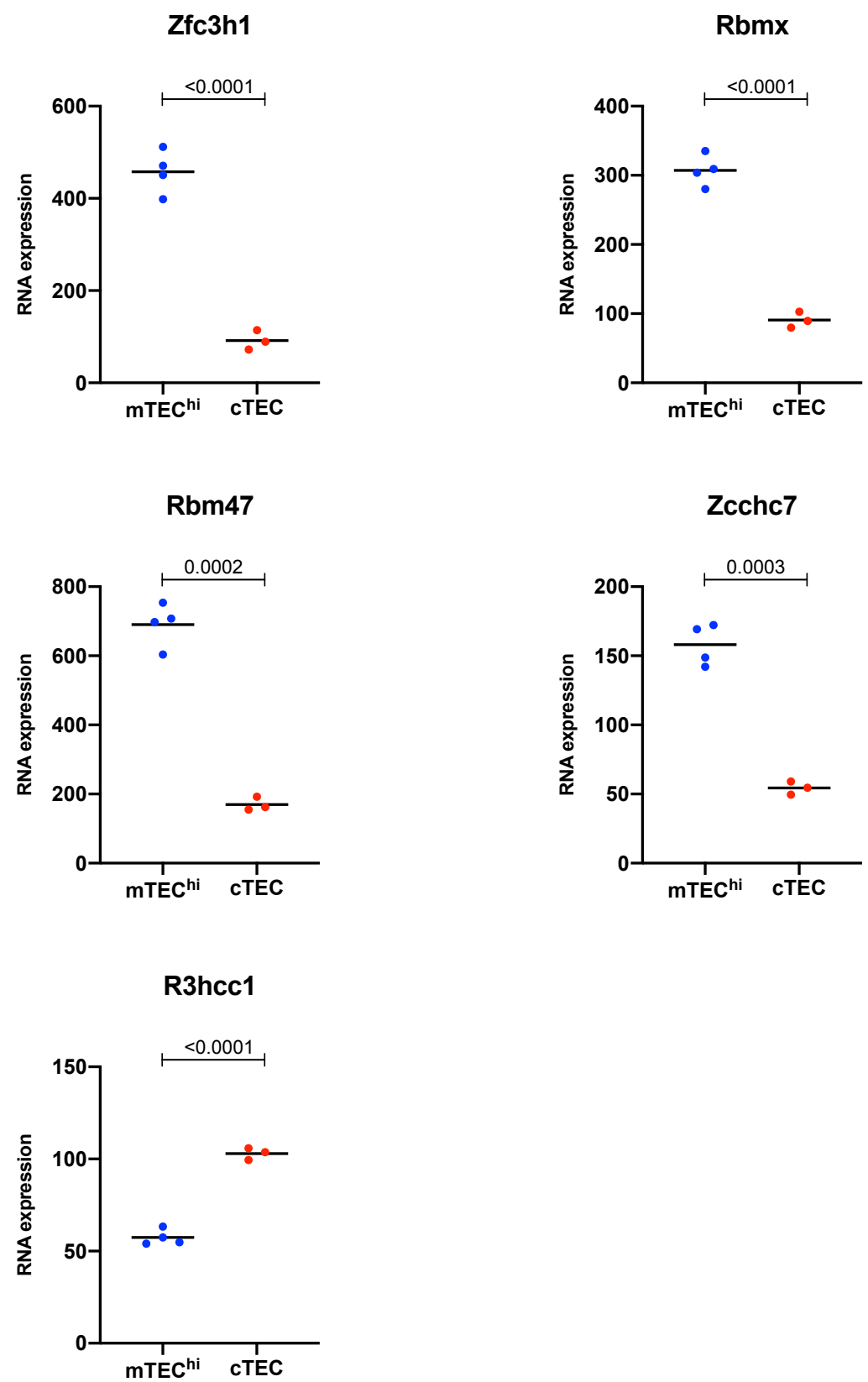

Figure 3: Multiple RNA-binding proteins uniquely define the $\mathrm{mTEChi}$ lineage and are among the most differentially expressed genes when comparing the mTEChi and cTEC transcriptomes.

The expression of each differentially expressed gene is represented graphically, with the mean RNA expression value denoted by a horizontal black bar: mTEChi, $n=4$; cTEC, $n=3$. The statistical significance of the difference between the mean values between each cell type was calculated using a two-tailed unpaired t-test with Welch's correction, shown as a $p$-value on each graph. 

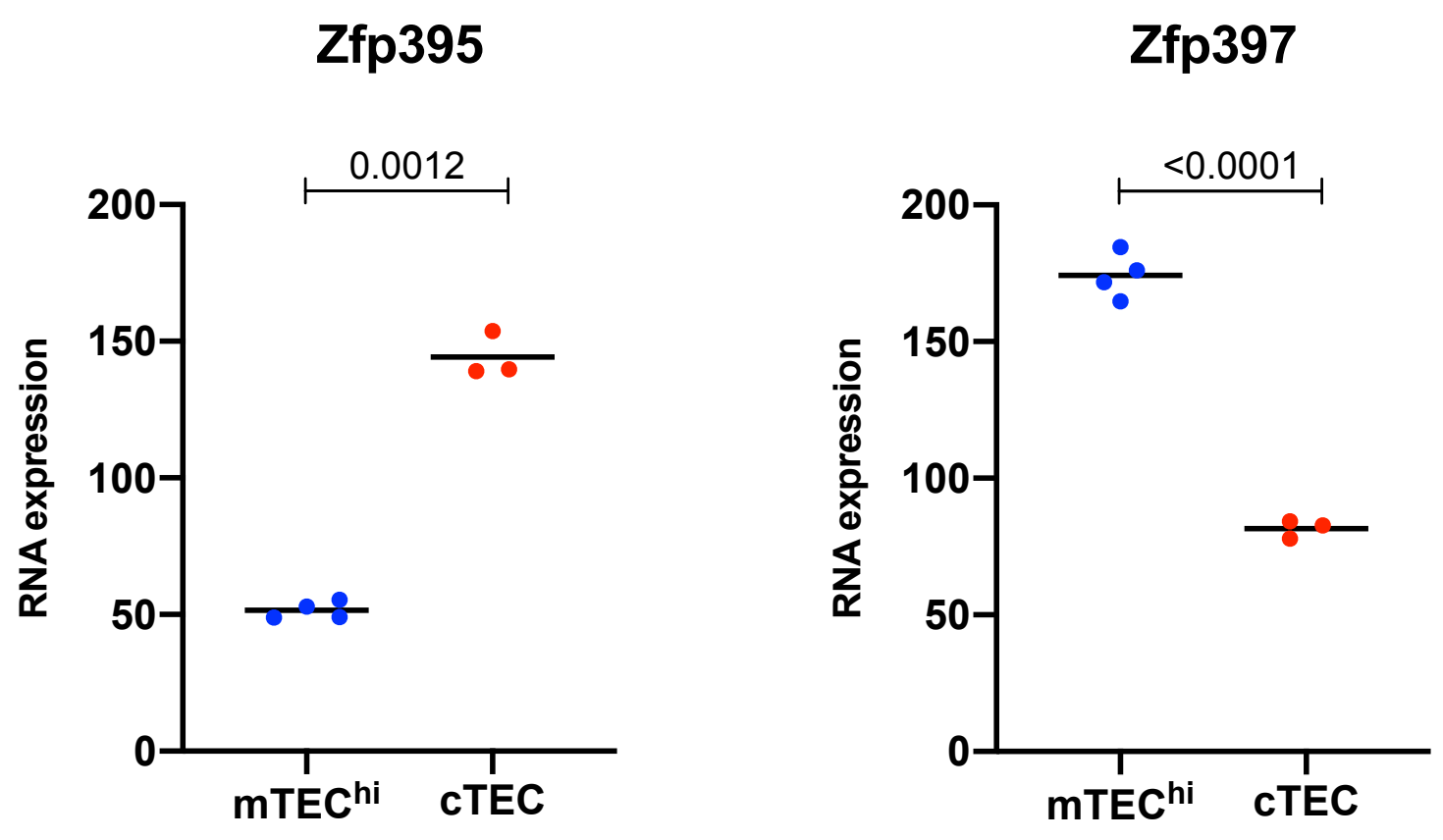

Figure 4: Two zinc-finger containing proteins uniquely define the $\mathrm{mTEChi}$ and cTEC lineages and are among the most differentially expressed genes when comparing the MTEChi and CTEC transcriptomes.

The expression of each differentially expressed gene is represented graphically, with the mean RNA expression value denoted by a horizontal black bar: mTEChi $n=4$; cTEC, $n=3$. The statistical significance of the difference between the mean values between each cell type was calculated using a two-tailed unpaired t-test with Welch's correction, shown as a $p$-value on each graph. 


\section{Table 1}

\begin{tabular}{|c|c|c|c|c|c|c|}
\hline Rank & p-value & $\mathbf{t}$ & B & Gene Symbol & Gene Name & ID \\
\hline 2 & $2.42 \mathrm{E}-08$ & -40.141 & -3.31 & Utf1 & undifferentiated embryonic cell transcription factor 1 & 10558580 \\
\hline 12 & $9.17 \mathrm{E}-07$ & 21.421 & -3.32 & Foxe1 & forkhead box E1 & 10504728 \\
\hline 17 & $1.22 \mathrm{E}-06$ & 20.386 & -3.33 & Zfp395 & zinc finger protein 395 & 10415991 \\
\hline 26 & $1.51 \mathrm{E}-06$ & -19.653 & -3.33 & Fezf2 & Fez family zinc finger 2 & 10417620 \\
\hline 35 & $2.57 \mathrm{E}-06$ & -17.905 & -3.33 & Zfp397 & zinc finger protein 397 & 10454298 \\
\hline 54 & 4.37E-06 & -16.324 & -3.34 & Med12I & mediator complex subunit 12 -like & 10492231 \\
\hline 61 & 5.03E-06 & -15.927 & -3.34 & $\mathrm{Rbmx}$ & RNA binding motif protein, $\mathrm{X}$ chromosome & 10604735 \\
\hline 64 & 5.19E-06 & 15.836 & -3.34 & Hoxd11 & homeobox D11 & 10472953 \\
\hline 74 & $6.08 \mathrm{E}-06$ & -15.406 & -3.35 & Rbpj & recombination signal binding protein for immunoglobulin kappa $\mathrm{J}$ region & 10521913 \\
\hline 76 & $6.27 \mathrm{E}-06$ & -15.324 & -3.35 & Baz2a & bromodomain adjacent to zinc finger domain, $2 \mathrm{~A}$ & 10367122 \\
\hline 92 & 8.33E-06 & -14.577 & -3.35 & $\mathrm{Rbm} 47$ & RNA binding motif protein 47 & 10530269 \\
\hline 125 & $1.16 \mathrm{E}-05$ & -13.755 & -3.36 & Sirt1 & sirtuin 1 & 10369738 \\
\hline 137 & $1.3 \mathrm{E}-05$ & 13.475 & -3.36 & R3hcc1 & $\mathrm{R} 3 \mathrm{H}$ domain and coiled-coil containing 1 & 10421188 \\
\hline 148 & $1.44 \mathrm{E}-05$ & -13.240 & -3.36 & Zfc3h1 & zinc finger, $\mathrm{C} 3 \mathrm{H} 1$-type containing & 10366409 \\
\hline 152 & $1.5 \mathrm{E}-05$ & 13.146 & -3.36 & Ascl4 & achaete-scute family bHLH transcription factor 4 & 10365469 \\
\hline 172 & $1.81 \mathrm{E}-05$ & -12.707 & -3.37 & Arid4a & AT rich interactive domain $4 \mathrm{~A}$ (RBP1-like) & 10396205 \\
\hline 205 & $2.34 \mathrm{E}-05$ & -12.149 & -3.37 & Myb///Myb & myeloblastosis oncogene///myeloblastosis oncogene & 10368199 \\
\hline 221 & $2.6 \mathrm{E}-05$ & -11.925 & -3.38 & Zcchc7 & zinc finger, $\mathrm{CCHC}$ domain containing 7 & 10504491 \\
\hline
\end{tabular}

Table 1: Transcription factors, epigenetic molecules, DNA and RNA binding factors that are among the most differentially expressed genes when comparing the transcriptomes of $M T E C h i$ and cTEC.

Rank order with respect to differential expression globally, $\mathrm{p}$-value, $\mathrm{t}$ (a moderated t-statistic), $B$, the log-odds that the gene is differentially expressed between each group, gene symbol, gene name and ID are listed (all provided by NCBI GEO2R). 